\title{
Stretchable Electronics Based on Laser Structured, Vapor Phase Polymerized PEDOT/Tosylate
}

\author{
Zaid Aqrawe $^{1, *}$, Christian Boehler ${ }^{2}{ }^{(D}$, Mahima Bansal ${ }^{3}\left(\mathbb{D}\right.$, Simon J. O’Carroll ${ }^{1}(\mathbb{D}$, \\ Maria Asplund ${ }^{2,4}$ and Darren Svirskis ${ }^{3}$ (D) \\ 1 Department of Anatomy \& Medical Imaging, School of Medical Sciences, University of Auckland, \\ Auckland 1023, New Zealand; s.ocarroll@auckland.ac.nz \\ 2 Department of Microsystems Engineering (IMTEK) and BrainLinks-BrainTools Center, University of \\ Freiburg, 79110 Freiburg, Germany; christian.boehler@imtek.de (C.B.); \\ maria.asplund@imtek.uni-freiburg.de (M.A.) \\ 3 School of Pharmacy, University of Auckland, Auckland 1023, New Zealand; \\ m.bansal@auckland.ac.nz (M.B.); d.svirskis@auckland.ac.nz (D.S.) \\ 4 Division of Nursing and Medical Technology, Luleå University of Technology, 97187 Luleå, Sweden \\ * Correspondence: zaqr001@aucklanduni.ac.nz or zaid.aqrawe@gmail.com
}

Received: 18 June 2020; Accepted: 21 July 2020; Published: 25 July 2020

\begin{abstract}
The fabrication of stretchable conductive material through vapor phase polymerization of poly(3,4-ethylenedioxythiophene) (PEDOT) is presented alongside a method to easily pattern these materials with nanosecond laser structuring. The devices were constructed from sheets of vapor phase polymerized PEDOT doped with tosylate on pre-stretched elastomeric substrates followed by laser structuring to achieve the desired geometrical shape. Devices were characterized for electrical conductivity, morphology, and electrical integrity in response to externally applied strain. Fabricated PEDOT sheets displayed a conductivity of $53.1 \pm 1.2 \mathrm{~S} \mathrm{~cm}^{-1}$; clear buckling in the PEDOT microstructure was observed as a result of pre-stretching the underlying elastomeric substrate; and the final stretchable electronic devices were able to remain electrically conductive with up to $100 \%$ of externally applied strain. The described polymerization and fabrication steps achieve highly processable and patternable functional conductive polymer films, which are suitable for stretchable electronics due to their ability to withstand externally applied strains of up to $100 \%$.
\end{abstract}

Keywords: conducting polymers; vapor phase polymerization; PDMS; elastomeric

\section{Introduction}

Stretchable electronics have become a topic of focus for many academic and industrial research groups due to their ability to enable myriad emerging applications such as skin-mounted sensors, biosensors, soft robotics, and wearable displays $[1,2]$. The challenge in fabricating stretchable electronics resides within the inherent un-stretchable nature of commonly used metallic films, such as gold, which are known to fracture and fail electrically at strains of 1-2\% [3] in both free-standing and polymer-supported states. Following approximately two decades of research, three main methods have been developed to achieve stretchable electronic materials: (i) engineering the geometry of rigid electrically conductive materials; (ii) the creation of a composite material with conductive fillers embedded into an elastomeric matrix; and (iii) the fabrication of intrinsically conductive materials [2]. Geometric engineering, employed by a number of research groups, has proven to impart stretchability onto rigid electrically conductive materials through the utilization of buckled or waved architectures. This method was initially described by Lacour et al. [4] and Jones et al. [5] in 2003 who discovered that deposition of thin layers $(<100 \mathrm{~nm})$ of chromium and gold onto pre-stretched elastomeric silicone 
substrates would create buckled structures in the metals nanostructure and enabled devices to withstand up to $26 \%$ strain without electrical failure, in a reproducible manner. The rationale behind these designs is that strain energy is dissipated through the straightening of buckled structures created through pre-stretching, rather than the electrical material itself. To build on this approach, interconnects can also be patterned into waved geometries on a macroscopic level to further contribute to the dissipation of strain upon stretching. In the literature, numerous waved geometries have been proposed, such as serpentine [6-11], horseshoe [12,13], kirigami [14], island [15], spiral [16,17], and fractal interconnects [18], which all serve to increase the maximum strain a device can withstand without failing electrically. To date, most of these devices which employ geometric engineering have been built with evaporated metallic films, such as gold and platinum (with their respective adhesion layers). The use of these materials is warranted due to their inertness, biocompatibility, and high conductivity. However, the difficulty that is associated with their functionalization for different applications and industries has drawn researchers to organic conductors, such as conductive polymers (CPs) [1,2,19]. CPs show great promise for stretchable electronics, due to their electronic tuneability by method of synthesis, ease of functionalization, and solution processability [20]—opening further avenues of deposition onto elastomeric substrates [21]. The most commonly employed CPs for stretchable electronics are cast from a commercially available aqueous dispersion of poly(3,4-ethylenedioxythiophene) (PEDOT) with poly(4-styrenesulfonate) (PSS) (PEDOT:PSS). Stretchable versions of PEDOT:PSS have been fabricated through geometric engineering [22], composite formation [2], and rendering the PEDOT:PSS to be intrinsically stretchable with additives [1]. To add to this rich body of literature, this article aims to arm readers with a novel approach of $\mathrm{CP}$ deposition for stretchable electronics though vapor phase polymerization (VPP), rather than solution casting of commercially available PEDOT:PSS films. VPP is a versatile method of achieving highly conductive, patternable $[23,24]$ and thin $C P$ films with easily tunable physical and electronic properties $[21,24,25]$. This communication aims to investigate the placement of VPP PEDOT films onto pre-stretched elastomeric substrates and the implications of this method on creating a stable stretchable electronic device. Furthermore, we introduce a method to laser structure VPP films in order to easily enable the patterning of multiple geometries of interest. The successful creation of a stable stretchable device which can be easily patterned and modified will open up avenues to a myriad of applications in fields such as bioelectronics, enabling the creation of microelectrode arrays that can study and help understand stretch-based injuries, for example traumatic brain injury or spinal cord injury. Furthermore, the versatility of this method allows for the ability to research various chemical additives and morphological structure and assess the impact these changes have on further optimization of this technology.

\section{Materials and Methods}

\subsection{Vapor Phase Polymerization of PEDOT/Tosylate}

The polymerization of PEDOT through vapor phase polymerization was carried out on a silicon wafer substrate. The wafer was first cleaned with isopropanol and then air dried using compressed air. An oxidant solution was prepared and comprised poly(ethylene glycol)-block-poly(propylene glycol)-block-poly(ethylene glycol) (PEG-PPG-PEG, 23\% w/w, Sigma-Aldrich Chemie GmbH, Germany), iron (III) tosylate (FeTOS, $15.4 \%$ w/w, Sigma-Aldrich Chemie GmbH, Germany), and ethanol (61.5\% w/w, Carl Roth GmbH + Co.KG, Karlsruhe, Germany). This oxidant solution was spun onto the silicon substrate at a speed of $1500 \mathrm{rpm}$ for $20 \mathrm{~s}$, with an acceleration of $500 \mathrm{rpm} \mathrm{s}^{-1}$, followed by heat curing at $70{ }^{\circ} \mathrm{C}$ for $60 \mathrm{~s}$. These samples were then placed within a desiccator which contained $100 \mu \mathrm{L}$ of 3,4-ethylenedioxythiophene (EDOT, Sigma-Aldrich Chemie GmbH, Germany) monomer in a petri-dish at its base. Air was evacuated from the desiccator using a vacuum pump followed by placement of the system in an oven with a temperature of $40^{\circ} \mathrm{C}$. The formation of PEDOT doped with tosylate (PEDOT/TOS) through VPP was allowed to occur under these conditions for $3 \mathrm{~h}$ [21]. At the end of this process, the samples were removed from the desiccator and rinsed with ethanol to wash 
away unreacted EDOT and residual FeTOS. Sequential polymerization steps obtained free-standing PEDOT/TOS films that could be transferred onto an elastomeric substrate, which meant additional layers polymerized over the previous PEDOT/TOS layer through repetition of the above process.

\subsection{Transfer of PEDOT/TOS onto Elastomeric Substrate}

Elastomeric substrates were made at dimensions of $50 \mathrm{~mm} \times 50 \mathrm{~mm} \times 1 \mathrm{~mm}(\mathrm{w} \times 1 \times \mathrm{h})$ from poly(dimethyl siloxane) (PDMS, RTV MED 1000, APM Technica AG, Germany) using a custom made Teflon mold. PDMS was diluted with n-heptan (Carl Roth GmbH + Co.KG, Karlsruhe, Germany) at a ratio of 1:1 (v:v), poured into the mold and left to cure overnight in humid conditions. PDMS substrates were pre-stretched to varying degrees $(0 \%, 40 \%, 60 \%$, and $80 \%)$ of their original size using a custom-made stretching device and placed into a water bath. PEDOT/TOS films were removed from the silicon substrate in an ethanol bath; the free-standing film was then transferred to the water bath containing the pre-stretched PDMS at its base. A water bath was chosen for this transfer as the surface tension of the water was sufficient to hold the PEDOT/TOS films in a flat state on its surface. The stretching device containing pre-stretched PDMS was slowly raised out of the water; during this process, the overlying PEDOT/TOS film was picked up by the PDMS sample and "cast" onto the elastomeric substrate as it exited the water. The PDMS-PEDOT/TOS system was dried carefully with pressurized air and was then allowed to relax back to its non-stretched state.

\subsection{Patterning of PEDOT/TOS on Elastomeric Substrate}

A nanosecond laser (ACI Laser GmbH, Workstation Professional, Nohra, Germany) was used to pattern the conductive PEDOT/TOS into electronic tracts with a contact on each side to allow for resistance measurements while the system was put under varying degrees of strain. To efficiently etch PEDOT/TOS from the PDMS surface, the following parameters were used: laser power $=10 \%$ ( 0.4 Watts), speed $=100 \mathrm{~mm} \mathrm{~s}^{-1}$, frequency $=8 \mathrm{kHz}$, and pulse width $=3 \mu \mathrm{s}$. These parameters equate to a pulse power of $16.6 \mathrm{~W}$ and a pulse power density of $1.73 \times 10^{10} \mathrm{Wm}^{-2}$. Once the PEDOT/TOS was structured, the PDMS was cut into individual devices with dimensions of $7 \mathrm{~mm} \times 20 \mathrm{~mm}$, each containing one PEDOT/TOS tract (track dimensions of $500 \mu \mathrm{m} \times 2000 \mu \mathrm{m}$ ).

\subsection{Conductivity Measurements}

Surface electrical conductivity of the fabricated PEDOT/TOS films was determined at room temperature using a four-point linear probe set up with $1.0 \mathrm{~mm}$ tip separation. A benchtop multi-meter (Hewlett Packard 34401A, Boeblingen, Germany) was used to measure resistivity across 0.02 " tungsten probes (72T-J3, American Probe \& Technologies Inc., Merced, CA, USA). A constant current was applied on the two outer electrodes, with the voltage drop across the two inner electrodes being measured. Measurements were made on three different locations across the film, and this was repeated on each subsequent polymerization layer. This process was done across three different PEDOT/TOS samples to obtain a triplicate conductivity value.

\subsection{Strain vs. Resistivity Measurements}

To measure resistance of the PDMS-PEDOT/TOS device while under strain, a custom stretch system was assembled (Figure 1). This system consisted of two moveable alignment chucks secured side by side on a large base plate. The PDMS-PEDOT/TOS was positioned so that one contact was on one chuck and the other contact resided on the other chuck, with the middle (or tract section) freestanding between the two chucks. Copper boards were then placed on top of the PDMS-PEDOT/TOS contacts and tightly bolted down. The board served two purposes: (i) to provide an electrical connection; and (ii) to mechanically anchor the contacts onto the chucks. To stretch the sample, one chuck was locked into a fixed position and the other was slowly moved further away using an in-built positioning wheel. The stage was moved $0.25 \mathrm{~mm}$ each time and a two-electrode resistance measurement was carried 
out at each strain interval. The resistance measurement was taken for $10 \mathrm{~s}$ and the average value was calculated from this trace.

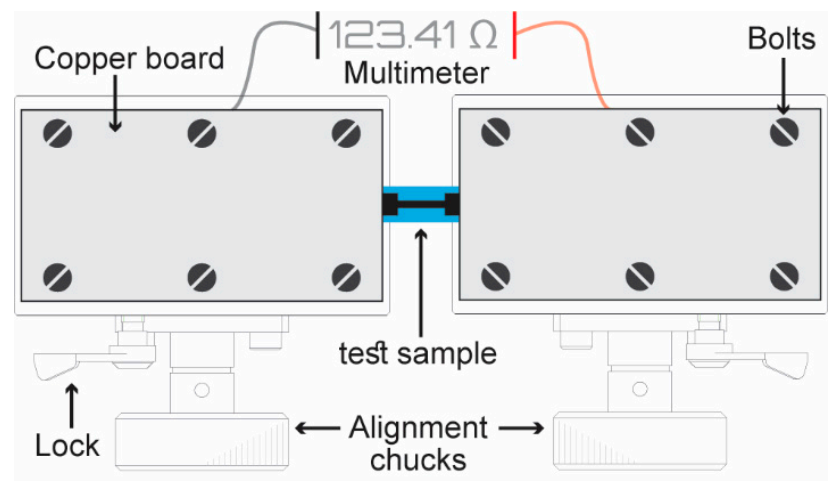

Figure 1. Stretch setup utilized which allows simultaneous resistivity measurements while the sample is put under strain.

\section{Results and Discussion}

PEDOT/TOS was successfully polymerized onto a silicon wafer using a multi-layer approach via sequential vapor phase polymerization steps. Table 1 highlights the conductivity measured at each layer number using a four-point conductivity measurement. A steady increase can be noticed with the addition of each sequential layer, indicating inter-layer as well as intra-layer conductivity. This trend is consistent with other reports of layered PEDOT deposition [26] and is thought to arise due to the multiple parallel PEDOT-rich pathways, which form a more pronounced electrical network that increases conductivity [27]. To further improve conductivity, conditions to control the VPP process temperature and pressure more accurately can be implemented, such as utilization of a vacuum oven. Previous experiments carried out in a vacuum oven achieved a conductivity of $1840 \pm 50 \mathrm{~S} \mathrm{~cm}^{-1}$ for one layer of PEDOT/TOS [21].

Table 1. Conductivity associated with number of PEDOT/TOS layers.

\begin{tabular}{cc}
\hline Layer Number & Conductivity $\left(\mathbf{S ~ c m}^{\mathbf{- 1}}\right)$ \\
\hline 1 & $37.7 \pm 1.4$ \\
2 & $43.2 \pm 2$ \\
3 & $53.1 \pm 1.2$ \\
\hline
\end{tabular}

At the third sequential polymerization layer, the PEDOT/TOS had enough mechanical stability to peel off from the silicon substrate and form a free-standing film in water or ethanol and this was taken as the number of layers employed for the stretchable electronic devices. In a previous publication, the thickness of five PEDOT/TOS layers prepared by a similar VPP process was determined to be $1.66 \pm 0.06 \mu \mathrm{m}$, with an unremarkable surface morphology; therefore, it is estimated that the total thickness of these films resides around $1 \mu \mathrm{m}$ [21]. PEDOT/TOS films were successfully transferred to pre-stretched PDMS substrates and structured to the desired pattern using a nano-second laser (Figure 2A). When examining the microstructure of these films, buckling can clearly be seen perpendicular to the stretch direction and is as a result of laying down the conductive PEDOT/TOS films onto pre-stretched PDMS that is then allowed to relax (Figure 2C-E). 


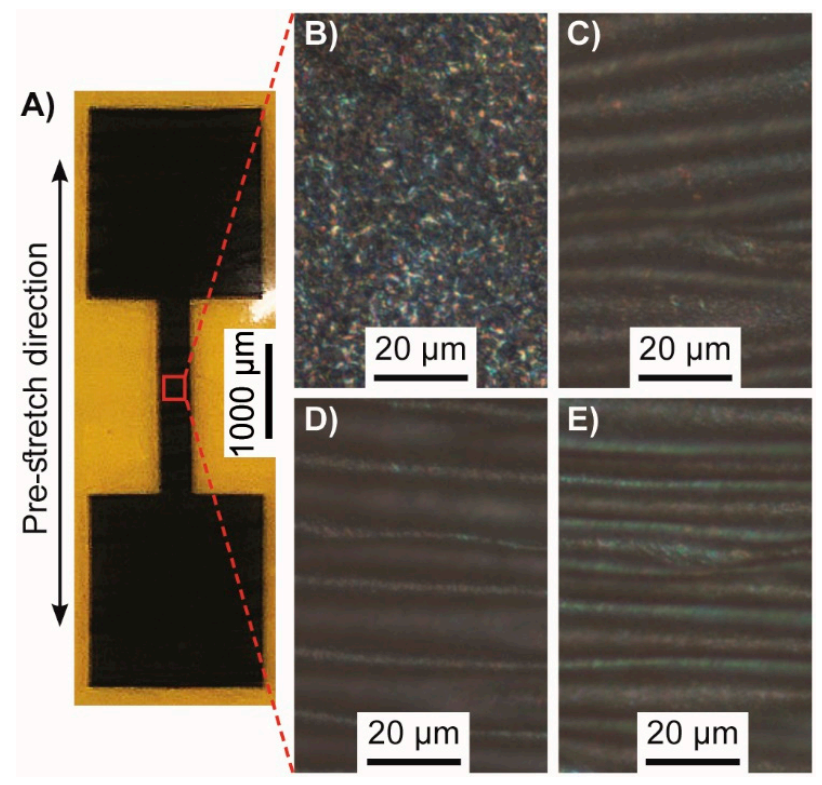

Figure 2. (A) Final device design with two contact pads joined by a $500 \mu \mathrm{m}$ wide by $2000 \mu \mathrm{m}$ long track. The device shown illustrates a laser structured PEDOT/TOS film on PDMS which was pre-stretched by $80 \%$. The device is clear, however, here it was imaged on a Kapton substrate, giving it a yellow color. The close-up images show the structure of PEDOT/TOS on PDMS which had: (B) $0 \%$ pre-stretch; (C) 40\% pre-stretch; (D) 60\% pre-stretch; and (E) 80\% pre-stretch. Clear buckled structures can be seen in PEDOT/TOS films which were placed onto pre-stretched PDMS that was then allowed to relax.

The strain vs. change in resistance profile $(\Delta R)$ of varying pre-stretched PEDOT/TOS samples is displayed in Figure 3. A sharp increase in $\Delta \mathrm{R}$ signified electrical disruption at the corresponding applied external strain. Analysis of this graph highlights the dependency of degree of pre-stretch on the maximum strain load that can be handled. In other words, the higher is the pre-stretch, the more functional strain the device can accommodate when in use. At $0 \%$ pre-stretch, the PEDOT films fail electrically after the first incremental strain step of $2 \%$, demonstrating the low intrinsic stretchability of the PEDOT/TOS material. Strategies to improve the intrinsic stretchability of PEDOT:PSS films have been highlighted in previous studies and could also be implemented in future works to further improve the performance of these PEDOT/TOS devices. The process of blending PEDOT:PSS with additives such as small molecule plasticizers, e.g., glycerol [28] and ionic liquids [29], has been reported and allows the PEDOT films to be stretched up to $50 \%$ without any detrimental effect on electrical properties [1]. In a similar fashion, the use of polymer additives such as poly(ethylene glycol) (PEG) [30], poly(ethylene oxide) (PEO) [30], poly(ethylene glycol)-block-poly(propylene glycol)-block-poly(ethylene glycol) triblock copolymer (PEO-PPO-PEO) [31], and poly(vinyl alcohol) [1] in the PEDOT matrix supported strain values of up to $100 \%$; however, one disadvantage is the decrease in electronic conductivity that arises upon addition of insulating polymeric materials. It should be noted that the current mixture used for VPP polymerization contains PEG-PPG-PEG, which is also theorized to impart some stretchable properties to the $\mathrm{CP}$ film, and further optimization of this mixture could provide more favorable intrinsic stretchability in future studies. Increasing the pre-stretch to $20 \%$ yielded a higher tolerance to externally applied strain, where the films failed at an external strain of above $20 \%$. At higher pre-stretches of $60 \%$ and $80 \%$, the films withstood externally applied strains of $80 \%$ and $100 \%$, respectively, before electrical disruption. An interesting observation for all pre-stretched samples was the return of an electrical path once the samples were allowed to relax after a sharp increase in resistance, highlighting the presence of compensatory conductive pathways. It should be noted that there is a mismatch in resistance of these "relaxed" devices when compared to pristine ones and this is likely to occur as a result of conductive path breakages during the stretch cycle; nonetheless, these data highlight the ability of these materials to maintain electrical conductivity even after their 
respective maximum strain limits are reached. It is again apparent that PEDOT/TOS subjected to greater pre-stretch $(>60 \%)$ produced a more stable system through observation of the relax phase which shows that the resistance returns closer to pristine levels when compared to a pre-stretch of $40 \%$. This may be explained by the way strain is dissipated in CPs subjected to greater pre-stretch, whereby breakages or disconnects occur in different ways. These data indicate the ability of the introduced buckled structures (Figure 2E) to successfully dissipate the strain energy and create functioning stretchable electronics through the utilization of VPP based PEDOT/TOS. It should be noted that, during these stretch/relax processes, the PEDOT/TOS did not delaminate from the underlying PDMS substrate in any of the samples tested indicating good adhesion between the two surfaces. Future in-depth mechanical testing is needed to quantitively assess the strength of this interface, such as the tape test to quantify strength of adhesion [32].
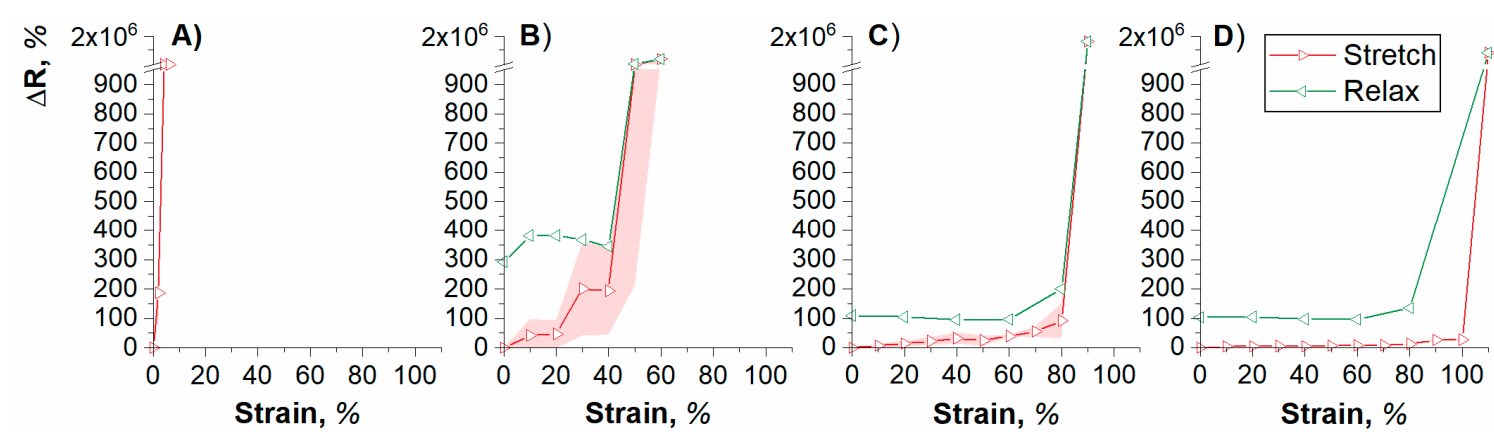

Figure 3. Strain vs. change in resistance profile of PDMS-PEDOT/TOS system under: (A) $0 \%$ pre-stretch; (B) $40 \%$ pre-stretch; (C) $60 \%$ pre-stretch; and (D) $80 \%$ pre-stretch. The graphs show both the stretching (red) and relaxing (green) cycles and the shaded areas indicate standard deviation $(n=3$, plotted as the average \pm SD).

\section{Conclusions}

The applicability of vapor phase polymerized PEDOT films for stretchable electronics is demonstrated, showing that these devices can withstand up to $100 \%$ strain under the right fabrication conditions. In addition, electrical pathways were partially restored upon relaxation of films, even after the maximum strain was surpassed. Further optimization of the polymerization process, and the introduction of additives to improve PEDOT film stretchability, are likely to further improve the performance of these devices. The ease of patterning through laser structuring was demonstrated and allows for many geometries to be tested using these techniques. It should be noted that, in our study, the conducting polymer is insulated only on one side and is fully exposed to the top. In certain applications, such as bioelectronics, a top insulating layer is needed to define electrode area and to protect the conducting polymer from factors such as humidity. Although not tested here, we believe this insulating layer could further help to mechanically stabilize the PEDOT layer as long as the bottom and top silicone layers could be put under equal tension. The myriad possibilities offered by vapor phase polymerization, and the ease of patterning through laser structuring described in this communication, opens doors for new research avenues in stretchable electronic devices.

Author Contributions: Conceptualization, Z.A., D.S., S.J.O., and M.A.; methodology, Z.A., C.B., and M.B.; formal analysis, Z.A.; resources, D.S. and M.A.; writing-original draft preparation, Z.A.; writing-review and editing, Z.A. and D.S.; supervision, D.S., M.A., and S.J.O.; project administration, Z.A.; and funding acquisition, Z.A. All authors have read and agreed to the published version of the manuscript.

Funding: This research was funded by the Neurological Foundation of New Zealand, grant number 1725-OCF, and the Health Research Council of New Zealand, grant number 19/007.

Acknowledgments: We acknowledge the researchers in the University of Freiburg, Microsystems Engineering, Biomedical Microsystems for their support and expertise.

Conflicts of Interest: The authors declare no conflict of interest. 


\section{References}

1. Kayser, L.V.; Lipomi, D.J. Stretchable Conductive Polymers and Composites Based on PEDOT and PEDOT:PSS. Adv. Mater. 2019, 31, 1806133. [CrossRef] [PubMed]

2. Wang, M.; Baek, P.; Akbarinejad, A.; Barker, D.; Travas-Sejdic, J. Conjugated polymers and composites for stretchable organic electronics. J. Mater. Chem. C 2019, 7, 5534-5552. [CrossRef]

3. Huang, H.; Spaepen, F. Tensile testing of free-standing $\mathrm{Cu}, \mathrm{Ag}$ and $\mathrm{Al}$ thin films and Ag/Cu multilayers. Acta Mater. 2000, 48, 3261-3269. [CrossRef]

4. Lacour, S.P.; Wagner, S.; Huang, Z.; Suo, Z. Stretchable gold conductors on elastomeric substrates. Appl. Phys. Lett. 2003, 82, 2404-2406. [CrossRef]

5. Jones, J.; Lacour, S.P.; Wagner, S.; Suo, Z. Stretchable wavy metal interconnects. J. Vac. Sci. Technol. A 2004, 22, 1723-1725. [CrossRef]

6. Widlund, T.; Yang, S.; Hsu, Y.Y.; Lu, N. Stretchability and compliance of freestanding serpentine-shaped ribbons. Int. J. Solids Struct. 2014, 51, 4026-4037. [CrossRef]

7. Yang, S.; Qiao, S.; Lu, N. Elasticity solutions to nonbuckling serpentine ribbons. J. Appl. Mech. Trans. ASME 2017, 84. [CrossRef]

8. Zhang, Y.; Fu, H.; Su, Y.; Xu, S.; Cheng, H.; Fan, J.A.; Hwang, K.C.; Rogers, J.A.; Huang, Y. Mechanics of ultra-stretchable self-similar serpentine interconnects. Acta Mater. 2013, 61, 7816-7827. [CrossRef]

9. Pan, T.; Pharr, M.; Ma, Y.; Ning, R.; Yan, Z.; Xu, R.; Feng, X.; Huang, Y.; Rogers, J.A. Experimental and Theoretical Studies of Serpentine Interconnects on Ultrathin Elastomers for Stretchable Electronics. Adv. Funct. Mater. 2017, 27. [CrossRef]

10. Sim, K.; Li, Y.; Song, J.; Yu, C. Biaxially Stretchable Ultrathin Si Enabled by Serpentine Structures on Prestrained Elastomers. Adv. Mater. Technol. 2019, 4. [CrossRef]

11. Huang, Y.; Mu, Z.; Feng, P.; Yuan, J. Mechanics Design for Compatible Deformation of Fractal Serpentine Interconnects in High-Density Stretchable Electronics. J. Appl. Mech. Trans. ASME 2019, 86. [CrossRef]

12. Li, M.; Xia, J.; Li, R.; Kang, Z.; Su, Y. Design of two-dimensional horseshoe layout for stretchable electronic systems. J. Mater. Sci. 2013, 48, 8443-8448. [CrossRef]

13. Ma, Q.; Zhang, Y. Mechanics of Fractal-Inspired Horseshoe Microstructures for Applications in Stretchable Electronics. J. Appl. Mech. Trans. ASME 2016, 83. [CrossRef]

14. Wang, Z.; Zhang, L.; Duan, S.; Jiang, H.; Shen, J.; Li, C. Kirigami-patterned highly stretchable conductors from flexible carbon nanotube-embedded polymer films. J. Mater. Chem. C 2017, 5, 8714-8722. [CrossRef]

15. Li, H.; Wang, Z.; Lu, S.; Ma, Y.; Feng, X. Elastomers with Microislands as Strain Isolating Substrates for Stretchable Electronics. Adv. Mater. Technol. 2019, 4. [CrossRef]

16. Lv, C.; Yu, H.; Jiang, H. Archimedean spiral design for extremely stretchable interconnects. Extrem. Mech. Lett. 2014, 1, 29-34. [CrossRef]

17. Qaiser, N.; Khan, S.M.; Nour, M.; Rehman, M.U.; Rojas, J.P.; Hussain, M.M. Mechanical response of spiral interconnect arrays for highly stretchable electronics. Appl. Phys. Lett. 2017, 111. [CrossRef]

18. Su, Y.; Wang, S.; Huang, Y.; Luan, H.; Dong, W.; Fan, J.A.; Yang, Q.; Rogers, J.A.; Huang, Y. Elasticity of fractal inspired interconnects. Small 2015, 11,367-373. [CrossRef]

19. Cuttaz, E.; Goding, J.; Vallejo-Giraldo, C.; Aregueta-Robles, U.; Lovell, N.; Ghezzi, D.; Green, R.A. Conductive elastomer composites for fully polymeric, flexible bioelectronics. Biomater. Sci. 2019, 7, 1372-1385. [CrossRef]

20. Boehler, C.; Aqrawe, Z.; Asplund, M. Applications of PEDOT in bioelectronic medicine. Bioelectron. Med. 2019, 2, 89-99. [CrossRef]

21. Bansal, M.; Sharma, M.; Bullen, C.; Svirskis, D. Free standing PEDOT films prepared by vapour phase polymerisation as electrically tuneable barriers to drug permeability. Mater. Sci. Eng. C 2018, 84, 248-253. [CrossRef] [PubMed]

22. Wen, Z.; Yang, Y.; Sun, N.; Li, G.; Liu, Y.; Chen, C.; Shi, J.; Xie, L.; Jiang, H.; Bao, D.; et al. A Wrinkled PEDOT:PSS Film Based Stretchable and Transparent Triboelectric Nanogenerator for Wearable Energy Harvesters and Active Motion Sensors. Adv. Funct. Mater. 2018, 28. [CrossRef]

23. Edberg, J.; Iandolo, D.; Brooke, R.; Liu, X.; Musumeci, C.; Andreasen, J.W.; Simon, D.T.; Evans, D.; Engquist, I.; Berggren, M. Patterning and Conductivity Modulation of Conductive Polymers by UV Light Exposure. Adv. Funct. Mater. 2016, 26, 6950-6960. [CrossRef] 
24. Brooke, R.; Edberg, J.; Iandolo, D.; Berggren, M.; Crispin, X.; Engquist, I. Controlling the electrochromic properties of conductive polymers using UV-light. J. Mater. Chem. C 2018, 6, 4663-4670. [CrossRef]

25. Mueller, M.; Fabretto, M.; Evans, D.; Hojati-Talemi, P.; Gruber, C.; Murphy, P. Vacuum vapour phase polymerization of high conductivity PEDOT: Role of PEG-PPG-PEG, the origin of water, and choice of oxidant. Polymer 2012, 53, 2146-2151. [CrossRef]

26. Greco, F.; Zucca, A.; Taccola, S.; Menciassi, A.; Fujie, T.; Haniuda, H.; Takeoka, S.; Dario, P.; Mattoli, V. Ultra-thin conductive free-standing PEDOT/PSS nanofilms. Soft Matter 2011, 7, 10642-10650. [CrossRef]

27. Takei, K. Flexible and Stretchable Medical Devices; Wiley: Hoboken, NJ, USA, 2018.

28. Zhou, J.; Li, E.Q.; Li, R.; Xu, X.; Ventura, I.A.; Moussawi, A.; Anjum, D.H.; Hedhili, M.N.; Smilgies, D.-M.; Lubineau, G.; et al. Semi-metallic, strong and stretchable wet-spun conjugated polymer microfibers. J. Mater. Chem. C 2015, 3, 2528-2538. [CrossRef]

29. Wang, Y.; Zhu, C.; Pfattner, R.; Yan, H.; Jin, L.; Chen, S.; Molina-Lopez, F.; Lissel, F.; Liu, J.; Rabiah, N.I.; et al. A highly stretchable, transparent, and conductive polymer. Sci. Adv. 2017, 3. [CrossRef]

30. Li, P.; Sun, K.; Ouyang, J. Stretchable and Conductive Polymer Films Prepared by Solution Blending. ACS Appl. Mater. Interfaces 2015, 7, 18415-18423. [CrossRef]

31. Lee, J.H.; Jeong, Y.R.; Lee, G.; Jin, S.W.; Lee, Y.H.; Hong, S.Y.; Park, H.; Kim, J.W.; Lee, S.S.; Ha, J.S. Highly Conductive, Stretchable, and Transparent PEDOT:PSS Electrodes Fabricated with Triblock Copolymer Additives and Acid Treatment. ACS Appl. Mater. Interfaces 2018, 10, 28027-28035. [CrossRef]

32. ASTM. Standard Test Methods for Rating Adhesion by Tape Test; ASTM International: West Conshohocken, PA, USA, 2017; Volume D3359-17.

(C) 2020 by the authors. Licensee MDPI, Basel, Switzerland. This article is an open access article distributed under the terms and conditions of the Creative Commons Attribution (CC BY) license (http://creativecommons.org/licenses/by/4.0/). 